\title{
TMT-Based Proteomic Analysis of Plasma from Children with Rolandic Epilepsy
}

\author{
Ji Sun, ${ }^{1}$ Tiechao Jiang, ${ }^{2}$ Feng Gu, ${ }^{3}$ Dihui Ma $\mathbb{D}^{4},{ }^{4}$ and Jianmin Liang $\mathbb{D}^{1}$ \\ ${ }^{1}$ Department of Pediatric Neurology, The First Hospital of Jilin University, Changchun, Jilin 130021, China \\ ${ }^{2}$ Department of Cardiology, The China-Japan Union Hospital of Jilin University, Changchun, Jilin 130031, China \\ ${ }^{3}$ Department of Infectious Diseases, The People's Hospital of Jilin Province, Changchun, Jilin 130021, China \\ ${ }^{4}$ Department of Neurology, The First Hospital of Jilin University, Changchun, Jilin 130021, China
}

Correspondence should be addressed to Dihui Ma; madihui@126.com and Jianmin Liang; jackyliangjm@163.com

Received 18 April 2020; Revised 5 September 2020; Accepted 16 September 2020; Published 7 October 2020

Academic Editor: Lance A. Liotta

Copyright (c) 2020 Ji Sun et al. This is an open access article distributed under the Creative Commons Attribution License, which permits unrestricted use, distribution, and reproduction in any medium, provided the original work is properly cited.

\begin{abstract}
Rolandic epilepsy is one of the most common epileptic syndromes in childhood. We used TMT-based proteomics and bioinformatics analysis to identify the differentially expressed proteins in plasma of children with Rolandic epilepsy. Our aim was to provide a molecular basis for exploring possible mechanisms underlying the pathogenesis of epilepsy. Subjects were divided into two groups (five in each): patients with Rolandic epilepsy as cases and patients with migraine as controls. Total proteins were extracted and quantitatively labeled with TMT, then analyzed using liquid chromatography mass spectrometry. Bioinformatics analysis was used to identify the hub genes. A total of 752 proteins were identified, of which 670 contained quantitative proteins. 217 differentially expressed proteins were identified, 46 of which were only upregulated in more than two groups and 111 of which were only downregulated in more than two groups. Bioinformatics analysis revealed top 10 hub genes in the up- and downregulated groups, respectively. Our study demonstrates that some differentially expressed proteins are associated with epilepsy. Activation of acute-phase or innate immune response and complement and fibrinogen systems and repression of glycolysis, lipoprotein metabolism, and antioxidant activity may play a role in the development of epilepsy.
\end{abstract}

\section{Introduction}

Rolandic epilepsy is the most common focal-onset epilepsy in childhood. The age at onset ranges from 3 to 13 years. It typically includes partial orofacial motor or sensory seizures, with or without secondary generalization, occurring most often during sleep or on awakening. Most cases of Rolandic epilepsy are idiopathic, but sometimes, they can be of symptomatic origin [1].

The diagnosis of epilepsy is mainly based on reliable clinical and historical features and EEG. However, the clinical and historical features sometimes are atypical, which make diagnosis difficult. Therefore, effective diagnostic tools and identification of disease biomarkers would be approved to facilitate correct clinical diagnosis as well as to generate treatments to prevent or cure epilepsy.
Plasma is the primary clinical specimen and represents the largest version of the human proteome present in any sample. It contains not only the classical "plasma proteins" but also all tissue proteins (as leakage markers) and numerous distinct immunoglobulin sequences. The human plasma proteome using multidimensional survey techniques has provided major challenges in proteomics during the past few years [2].

Nowadays, there have been few reports on the use of plasma proteome for seizure biomarker identification in children with Rolandic epilepsy. This was the reason why we performed this trial to discover a panel of diseasespecific biomarkers of Rolandic epilepsy from patients' plasma that seemed to contribute to illustrate the mechanisms of epileptogenesis and to be a useful tool for epilepsy diagnosis. 
TABLE 1: Clinical features of patients with Rolandic epilepsy and controls.

\begin{tabular}{lccccc}
\hline Number & Sex & Age (year) & Age of onset (year) & Duration (years) & VEEG/EEG \\
\hline Patients with Rolandic epilepsy & & & & SP (B) \\
E1 & M & $9.8 \mathrm{y}$ & 5 & 4 & SP (R) \\
E2 & $\mathrm{M}$ & $11 \mathrm{y}$ & 10 & 0.3 & SP (L) \\
E3 & $\mathrm{M}$ & $7.4 \mathrm{y}$ & 6 & 1 & SP (R) \\
E4 & $\mathrm{F}$ & $9 \mathrm{y}$ & 8 & 1 & SP (B) \\
E5 & $\mathrm{F}$ & $9.2 \mathrm{y}$ & 8 & 1 & VEEG/EEG \\
Number & Sex & Age (year) & Duration (years) & MRI & $\mathrm{n}$ \\
Controls & & & & $\mathrm{n}$ & $\mathrm{n}$ \\
C1 & $\mathrm{M}$ & $10 \mathrm{y}$ & 5 & $\mathrm{n}$ & $\mathrm{n}$ \\
C2 & $\mathrm{M}$ & $11 \mathrm{y}$ & 3 & $\mathrm{n}$ & $\mathrm{n}$ \\
C3 & $\mathrm{M}$ & $9 \mathrm{y}$ & 3 & $\mathrm{n}$ & $\mathrm{n}$ \\
C4 & $\mathrm{F}$ & $9 \mathrm{y}$ & 4 & $\mathrm{n}$ & $\mathrm{n}$ \\
C5 & $\mathrm{F}$ & $10 \mathrm{y}$ & 3 & & \\
\hline
\end{tabular}

M: male; F: female; R: right; L: left; B: Bilateral; EEG: electroencephalogram; VEEG: video electroencephalogram; SP: spike wave; n: normal; MRI: magnetic resonance imaging. E1-E5 represented five patients with Rolandic epilepsy; C1-C5 represented five patients with migraine.

\section{Materials and Methods}

2.1. Subjects. All the individuals including the epilepsy group and the control group were recruited from May 1, 2017, to Dec 1, 2018, at random. We labeled the patients with Rolandic epilepsy as E1 to E5 and the patients with migraine as C1 to C5. The epilepsy group consisted of five patients ranging from7 to 11 years old (mean $\pm \mathrm{SD}, 9.28 \pm 1.30$ years) with three men and two women. All patients underwent a comprehensive clinical examination, including a medical history, neurological examination, routine laboratory tests, EEG, and brain MRI. Epilepsy was diagnosed as Rolandic epilepsy according to the criteria proposed by the International League Against Epilepsy (ILAE) in 2001 [3] and had no underlying etiology. All patients had normal brain MRI and never taken any antiepilepsy drugs (AEDs).

The control group consisted of five sex- and age-matched individuals who were diagnosed as having migraine according to the criteria proposed by the International Headache Society (IHS) in 2018 [4]. The demographic and diseaserelated characteristics of the patients and controls are shown in Table 1. All people recruited in the research were of Chinese origin. Our hospital's Research Ethics Board approved this study (project identification code: 2017-295). Written informed consent was obtained from parents or guardians before recruitment.

\section{Protein Extraction and Identification}

3.1. Plasma Samples. For each subject, $5 \mathrm{ml}$ of venous blood samples was collected. Firstly, the blood samples were centrifuged at $2000 \times \mathrm{g}$ for $15 \mathrm{~min}$ at $4^{\circ} \mathrm{C}$ to collect the plasma, which was then subpackaged and stored at $-80^{\circ} \mathrm{C}$ until further analysis. Secondly, the cellular debris of the plasma sample was removed by centrifugation at $12000 \times \mathrm{g}$ for $10 \mathrm{~min}$ at $4^{\circ} \mathrm{C}$. Then, the supernatant was transferred to a new centrifuge tube. The top 12 high-abundance proteins were removed by Pierce $^{\mathrm{TM}}$ Top 12 Abundant Protein Depletion Spin Columns
Kit (ThermoFisher Scientific, Waltham, MA, USA). Finally, the protein concentration was determined with a BCA kit (Beyotime, Jiangsu, China) according to the manufacturer's instructions.

3.2. Trypsin Digestion. For digestion, the protein solution was reduced with $5 \mathrm{mM}$ dithiothreitol for $30 \mathrm{~min}$ at $56^{\circ} \mathrm{C}$ and alkylated with $11 \mathrm{mM}$ iodoacetamide for $15 \mathrm{~min}$ at room temperature in darkness. The protein sample was then diluted by adding $100 \mathrm{mM}$ TEAB to urea concentration less than $2 \mathrm{M}$. Finally, trypsin was added at 1:50 trypsin-to-protein mass ratio for the first digestion overnight and 1:100 trypsin-toprotein mass ratio for a second $4 \mathrm{~h}$ digestion.

3.3. TMT Labeling. After trypsin digestion, peptide was desalted by Strata-X C18 SPE column (Phenomenex, Torrance, CA, USA) and vacuum-dried. Peptide was reconstituted in $0.5 \mathrm{M}$ TEAB and processed according to the manufacturer's protocol for a TMT (Tandem Mass Tag) kit (ThermoFisher Scientific, Waltham, MA, USA). Briefly, one unit of TMT reagent was thawed and reconstituted in acetonitrile. The peptide mixtures were then incubated for $2 \mathrm{~h}$ at room temperature and pooled, desalted, and dried by vacuum centrifugation.

3.4. HPLC Fractionation. The tryptic peptides were fractionated into fractions by high-pH reverse-phase HPLC (high-performance liquid chromatography) using Agilent 300Extend C18 column ( $5 \mu \mathrm{m}$ particles, $4.6 \mathrm{~mm}$ ID, $250 \mathrm{~mm}$ length, Agilent, Santa Clara, CA, USA). Briefly, peptides were first separated with a gradient of $8 \%$ to $32 \%$ acetonitrile ( $\mathrm{pH} 9.0$ ) over $60 \mathrm{~min}$ into 60 fractions. Then, the peptides were combined into 18 fractions and dried by vacuum centrifuging.

3.5. LC-MS/MS Analysis. The tryptic peptides were dissolved in $0.1 \%$ formic acid (solvent A), directly loaded onto a homemade reversed-phase analytical column (15 cm length, $75 \mu \mathrm{m}$ ID). The gradient was comprised of an increase from $9 \%$ to $24 \%$ solvent B (0.1\% formic acid in $90 \%$ acetonitrile) over 
$38 \mathrm{~min}, 24 \%$ to $35 \%$ in $14 \mathrm{~min}$, and climbing to $80 \%$ in $4 \mathrm{~min}$ then holding at $80 \%$ for the last $4 \mathrm{~min}$, all at a constant flow rate of $700 \mathrm{nl} / \mathrm{min}$ on an EASY-nLC 1000 UPLC (ultraperformance liquid chromatography) system (ThermoFisher Scientific, Waltham, MA, USA). The peptides were subjected to NSI source followed by tandem mass spectrometry (MS/MS) in Q Exactive ${ }^{\mathrm{TM}}$ Plus (ThermoFisher Scientific, Waltham, MA, USA) coupled online to the UPLC. The electrospray voltage applied was $2.0 \mathrm{kV}$. The $\mathrm{m} / z$ scan range was 350 to 1800 for full scan, and intact peptides were detected in the Orbitrap at a resolution of 70,000. Peptides were then selected for MS/MS using NCE setting as 28, and the fragments were detected in the Orbitrap at a resolution of 17,500. A data-dependent procedure alternated between one MS scan and $20 \mathrm{MS} / \mathrm{MS}$ scans with $15.0 \mathrm{~s}$ dynamic exclusion. Automatic gain control (AGC) was set at 5E4. Fixed first mass was set as $100 \mathrm{~m} / z$.

3.6. Database Searching. The resulting MS/MS data were processed using the MaxQuant search engine (v.1.5.2.8). Tandem mass spectra were searched against the Swiss-Prot human database concatenated with a reverse decoy database. Trypsin/P was specified as a cleavage enzyme allowing up to 2 missing cleavages. The mass tolerance for precursor ions was set as $20 \mathrm{ppm}$ in the first search and $5 \mathrm{ppm}$ in the main search, and the mass tolerance for fragment ions was set as $0.02 \mathrm{Da}$. FDR was adjusted to $1 \%$.

\section{Bioinformatics Analysis}

Differentially expressed proteins with expression $\log 2$ fold change $>1.2$ and $p$ value $<0.05$ were selected as candidate proteins for further analysis. Then, Gene Ontology (GO) annotation proteome was derived from the UniProt-GOA database (http://www.ebi.ac.uk/GOA/). If some identified proteins were not annotated by the UniProt-GOA database, the InterProScan software would be used to annotate the protein's GO function based on a protein sequence alignment method. Proteins were classified by GO annotation into three categories: biological process, cellular compartment, and molecular function. For each category, a two-tailed Fisher exact test was employed to test the enrichment of the differentially expressed protein against all identified proteins. The GO with a corrected $p$ value $<0.05$ is considered significant. The KEGG (Kyoto Encyclopedia of Genes and Genomes, https://http://www.kegg.jp/) database was used to identify enriched KEGG pathways by a two-tailed Fisher exact test to test the enrichment of the differentially expressed protein against all identified proteins [5]. The results were filtered by the following criteria: a corrected $p$ value $<0.05$ and protein counts $>5$. The protein-protein interaction (PPI) networks of the differentially expressed proteins were established based on STRING (Search Tool for the Retrieval of Interacting Genes, https://string-db.org) [6]. We set confidence score $\geq 0.4$ and the maximum number of interactors $=0$ as the cutoff criterion. Then, the top 10 hub genes of the differentially expressed proteins were screened by the cytoHubba from the Cytoscape 3.7.0 (http://www .cytoscape.org/) platform according to the high degree of connectivity [7].

\section{Statistical Analysis}

All experimental data were presented as means \pm SD using SPSS software (version 13.0, SPSS).

\section{Results}

6.1. TMT-Based Quantitative Proteomic Basis Data Analysis and Overall Protein Identification. When plasma of patients with Rolandic epilepsy was compared with that of controls using TMT-based quantitative proteomics, a total of 213,300 $(17,617$ matched) spectra were obtained. Among these spectra, 4659 identified peptides (4541 unique peptides) and 752 identified proteins (670 quantified proteins) were detected (Table S1), and the average peptides mass error was $<10 \mathrm{ppm}$, indicating a high mass accuracy of the MS data (Figure S1a). The lengths of most identified peptides were 8 to 20 amino acid residues (Figure S1b), suggesting that our sampling met the required standard. The detailed information of identified proteins, including protein accession, protein description, gene name, protein molecular weight, peptide number, and matching scores, is shown in Table S2.

6.2. Identification of Differentially Expressed Proteins. Differentially expressed proteins (DEPs) were defined as those with a $>1.2$-fold change in relative abundance $(p<0.05)$ between five patients and controls, respectively. In total, 217 differentially expressed proteins were identified, 46 of which were only upregulated in more than two groups (patient vs. control) and 111 of which were only downregulated in more than two groups. The detailed information of differentially expressed proteins is shown in Table S3.

6.3. Enrichment Analysis of DEPs in GO. Above all, the significantly enriched GO terms of the biological process in the upregulated group were investigated. The top $12 \mathrm{GO}$ terms were immune or inflammatory response related proteins, further demonstrating the result that significant enrichment of the immune or inflammatory response pathway was observed during the initiation and development process of epilepsy [8-11]. In addition, the top 3 GO terms of the molecular function in the upregulated group were vitamin binding, complement binding, or RAGE receptor binding related proteins, indicating significant influence of vitamin and immunoglobulin superfamily on epilepsy control [1214]. The top 6 GO terms of the cellular component in the upregulated group were blood microparticle, membrane attack complex, pore complex, fibrinogen complex, and specific and secretory granule lumen related proteins, illustrating hyperactivation of the complement cascade and dysregulation of lipid and fibrinogen systems in epilepsy patients [1521](Figure 1(a)). Then, the significantly enriched GO terms of the biological process in the downregulated group were investigated. The top $5 \mathrm{GO}$ terms were oxidation-reduction and oxidative stress response related proteins, further demonstrating the important role of oxidative stress in epilepsy [22]. The top $7 \mathrm{GO}$ terms of the molecular function in the 
$-\log 10$ (Fisher's exact test $p$ value)

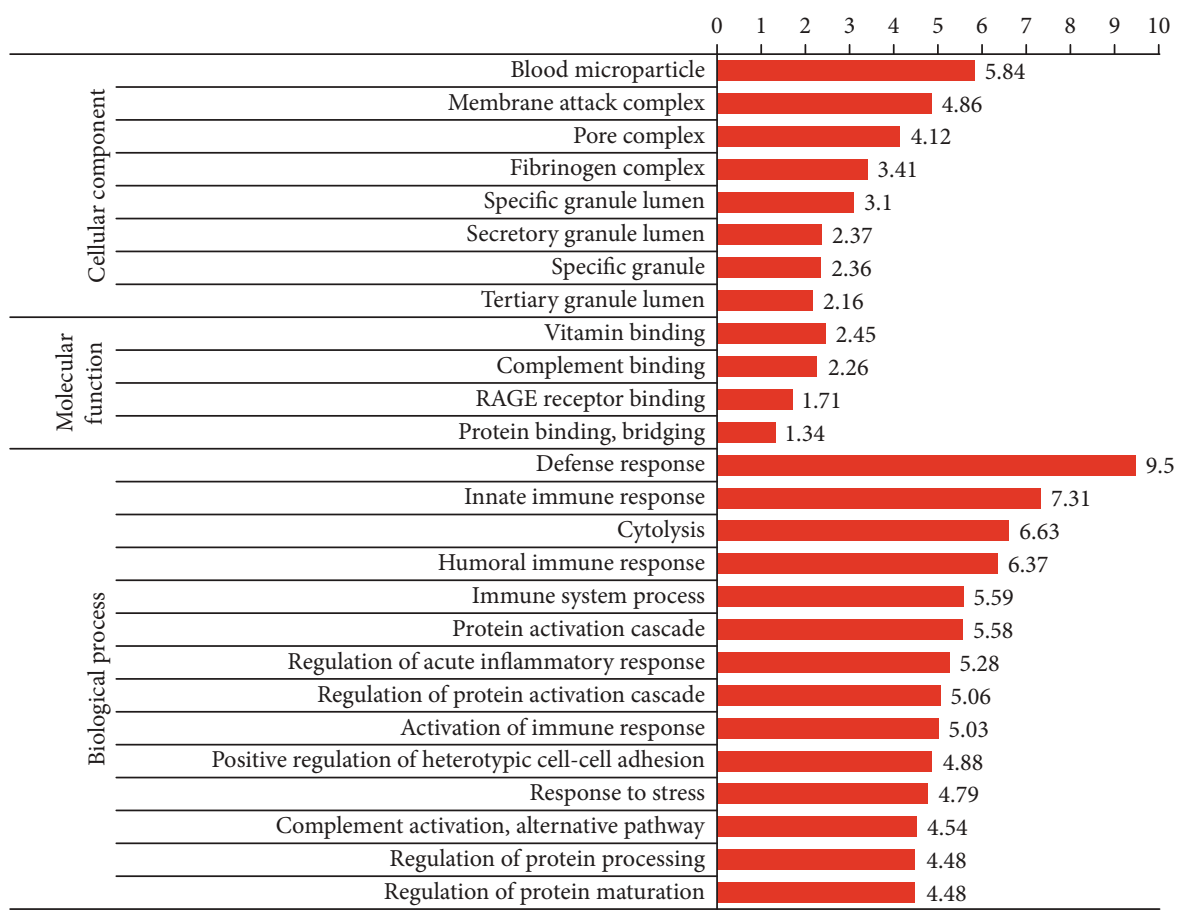

(a)

$-\log 10$ (Fisher's exact test $p$ value)

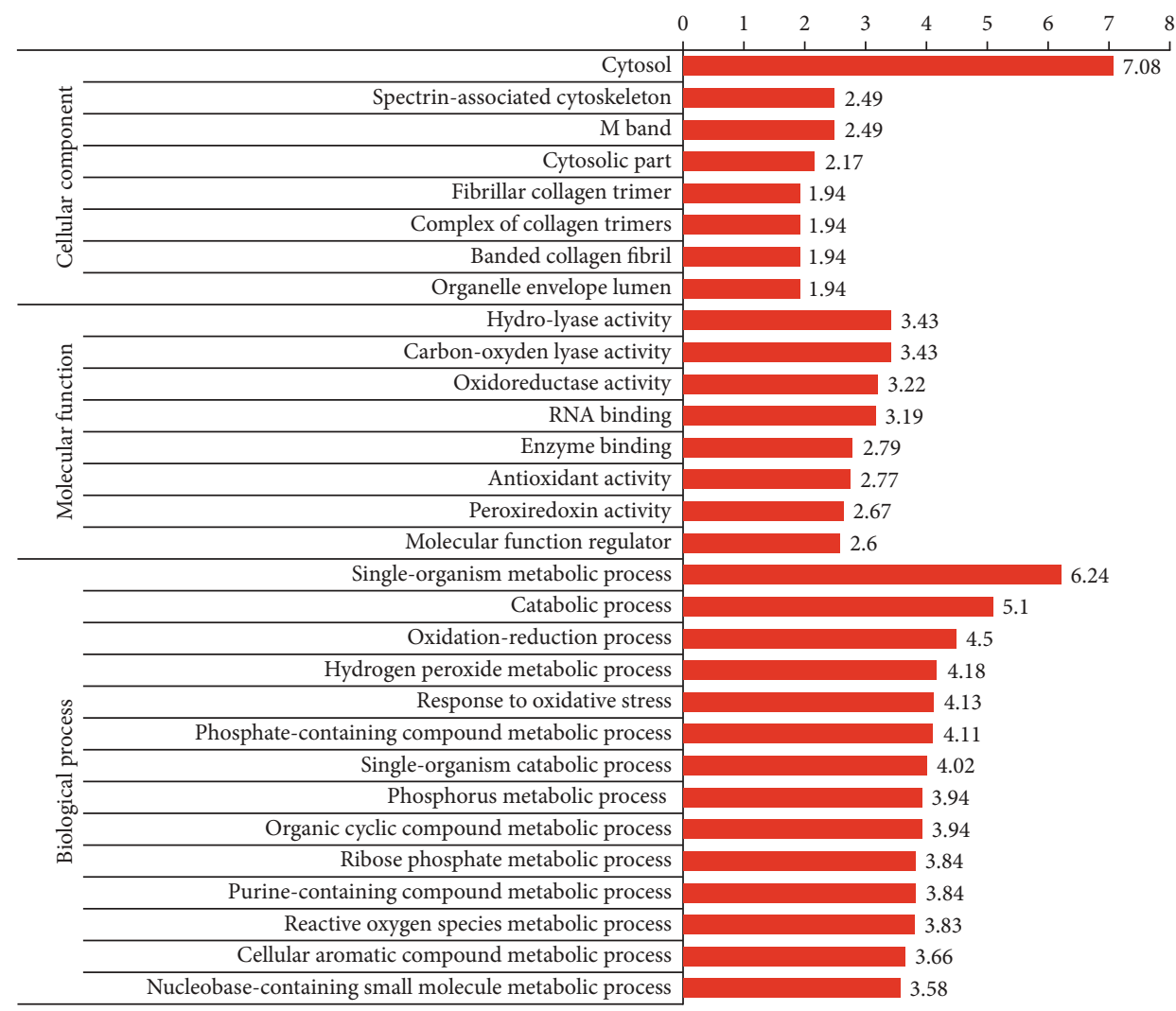

(b)

FIGURE 1: GO term analysis of differentially expressed proteins (DEPs): (a) GO terms of DEPs in the upregulated group; (b) GO terms of DEPs in the downregulated group. GO: Gene Ontology. 


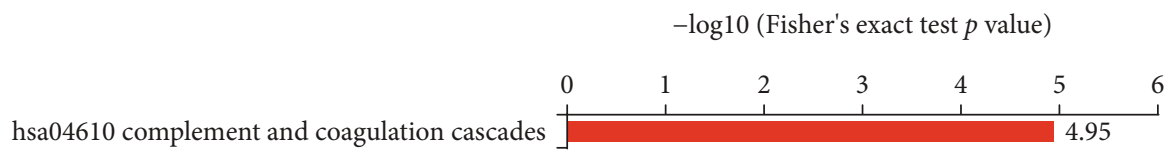

(a)

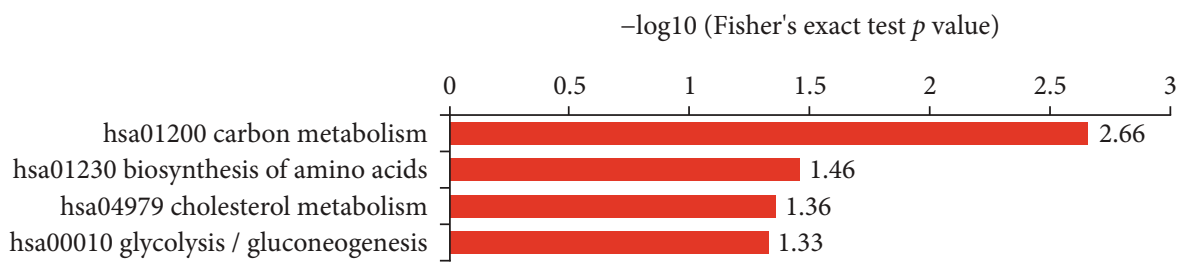

(b)

FIGURE 2: KEGG pathway enrichment analysis of DEPs: (a) pathway enrichment in the upregulated group; (b) pathway enrichment in the downregulated group. The pathway enrichment statistical analysis was performed by a two-tailed Fisher exact test. The $x$-axis is the $\log 10$ ( $p$ value). The $y$-axis is the enrichment pathway. KEGG: Kyoto Encyclopedia of Genes and Genomes.

downregulated group were oxidoreductase activity, antioxidant activity, and peroxiredoxin activity related proteins, indicating a significantly decreased level of antioxidant activity in the plasma of epilepsy patients. The top $4 \mathrm{GO}$ terms of the cellular component in the downregulated group were cytosol and cytosolic part related proteins, illustrating that most of the active proteins are present in the cytoplasm (Figure 1(b)).

6.4. Enrichment Analysis of DEPs in the KEGG Pathway. KEGG pathway enrichment analysis demonstrated that the complement and coagulation cascades were the most significantly affected pathways in the upregulated group. In addition, various metabolic pathways were significantly enriched in the downregulated group, of which were carbon metabolism, biosynthesis of amino acids, cholesterol metabolism, and glycolysis pathways (Figure 2). The results of the KEGG pathway analysis further indicated that hyperactivation of the complement cascade and dysregulation of lipid and glycometabolism systems are associated with the process of epilepsy development.

6.5. PPI Coexpression Network and the Top $10 \mathrm{Hub}$ Genes. The PPI networks of these DEPs in the up- and downregulated group are shown, respectively (Figure 3 ). Then, the top 10 hub genes were screened by cytoHubba according to the high degree of connectivity (Table 2). These hub genes were related with acute-phase or innate immune response, complement activation, regulation of fibrinogen systems, glycolysis, lipoprotein metabolism, and oxidative stress reaction.

\section{Discussion}

Rolandic epilepsy is an important and common disease in childhood. The cause of epilepsy remains unclear. In this study, we used a TMT-based quantitative proteomic analysis tool to detect the meaningful biomarkers in the plasma of Rolandic epilepsy patients. Here, we focus on hub genes in our study.
Fibronectin (FN1) was among the top 10 hub genes in the upregulated group which played an important role in maintaining cell morphology, intercellular adhesion, cell migration or chemotaxis, cell phagocytosis, hemostasis, damage repair, and body defense. Dixit et al. performed transcriptome analysis of hippocampal tissues resected from patients with MTLE-HS using the RNAseq approach. Differential gene expression analysis revealed that fibronectin was upregulated and was in the center of the network [23].

Alpha-1-acid glycoprotein (ORM) was also in the upregulated group which had many activities including, but not limited to, acting as an acute-phase reactant and disease marker, modulating immunity, binding and carrying drugs, maintaining the barrier function of capillary, and mediating the sphingolipid metabolism [24]. So far, there have been no reports on the relationship between alpha-1-acid glycoprotein and epilepsy.

Serum amyloid P-component (APCS) showed increase expression in the plasma of patients with epilepsy. Serum amyloid P-component belongs to the group of short pentraxins in the pentraxin family, which is known to be involved the humoral innate immune system spanning the complement system, inflammation, and coagulation [25]. Urbanyi et al. demonstrated that serum amyloid P-component induced neuronal apoptosis through penetrating the plasma membrane and translocating selectively into the nuclei of neurons [26]. But the relationship between serum amyloid P-component and epilepsy has not been known.

Fibrinogen (FGG, FGA) was also found to be increased in the plasma of patients with epilepsy. Hamed et al. reported that the level of fibrinogen was increased in the serum of adult patients with epilepsy compared with that of a healthy control, which demonstrated that vascular risk factor might be one of the independent predictors of asymptomatic atherosclerosis [27].

The complement system including complement component $\mathrm{C} 9$, complement component $\mathrm{C} 8$ beta chain, complement C4-B, and complement factor I was activated in the patient group, which was consistent with the result that the complement cascade was involved during epileptogenesis in the chronic epileptic phase in both experimental and human 


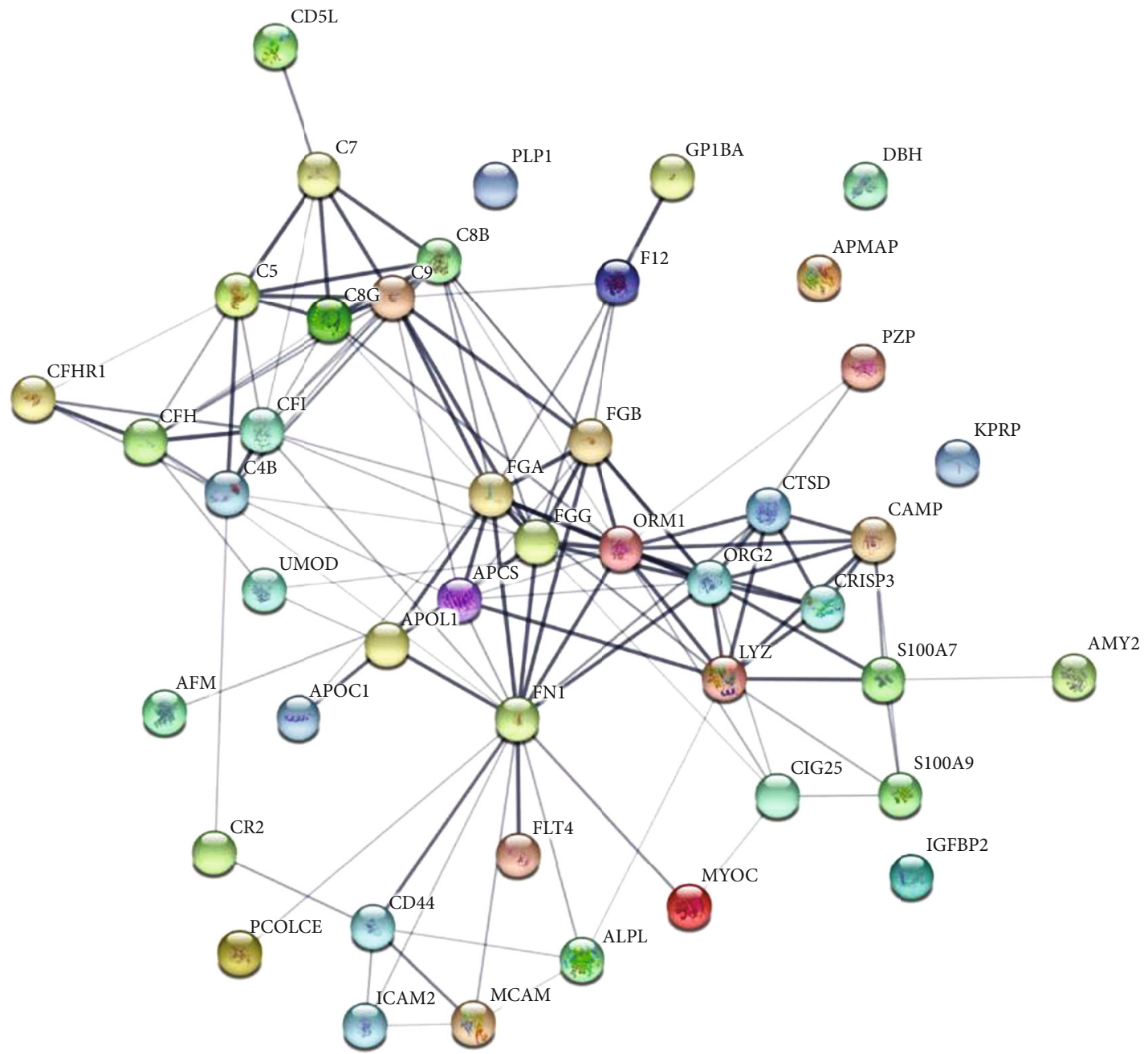

(a)

FIGURe 3: Continued. 


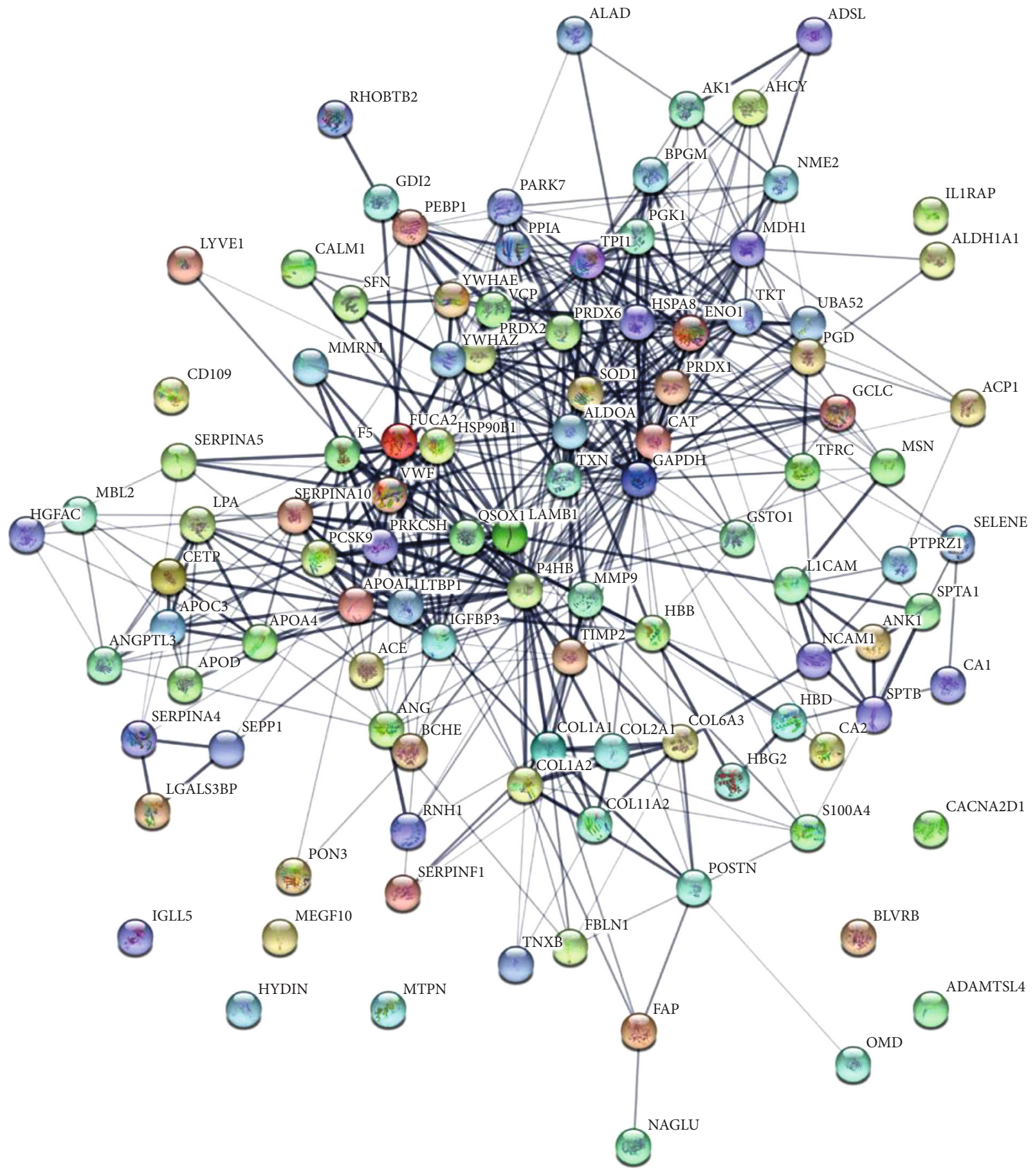

(b)

FIGURE 3: PPI coexpression network of DEPs: (a) PPI coexpression network in the upregulated group; (b) PPI coexpression network in the downregulated group.

TLE [15]. The persistence of complement activation may contribute to a sustained inflammatory response and destabilize neuronal networks involved.

Some glycolytic enzymes including glyceraldehyde3-phosphate dehydrogenase (GAPDH), triosephosphate isomerase (TPI1), phosphoglycerate kinase 1(PGK1), and fructose-bisphosphate aldolase A (ALDOA) play an impor- tant role in epileptogenesis. The decreased GAPDH kinase activity on $\gamma$-aminobutyric acid type $A$ receptors $\left(G A B A_{A} R\right)$ can reduce both endogenous phosphorylation and $G A B A_{A} R$ function. This dysfunction likely contributes to seizure generation and/or transition from the interictal to the ictal state [28]. In addition to its important catalytic role in glycolysis, ALDOA has other functions, such as signal transduction, 
TABLE 2: Top 10 hub genes in the upregulated group as well as top 10 hub genes in the downregulated group.

\begin{tabular}{|c|c|c|c|c|c|}
\hline \multicolumn{2}{|c|}{ Top 10 hub genes in upregulated group } & \multicolumn{4}{|c|}{ Top 10 hub genes in the downregulated group } \\
\hline Database identifier & Display name & Degree & Database identifier & Display name & Degree \\
\hline 9606.ENSP00000346839 & FN1 & 17 & 9606.ENSP00000229239 & GAPDH & 47 \\
\hline 9606.ENSP00000259396 & ORM1 & 16 & 9606.ENSP00000327801 & $\mathrm{P} 4 \mathrm{HB}$ & 36 \\
\hline 9606.ENSP00000394936 & ORM2 & 14 & 9606.ENSP00000234590 & ENO1 & 30 \\
\hline 9606.ENSP00000336829 & FGG & 13 & 9606.ENSP00000229270 & TPI1 & 27 \\
\hline 9606.ENSP00000306361 & FGA & 13 & 9606.ENSP00000362413 & PGK1 & 25 \\
\hline 9606.ENSP00000263408 & C9 & 12 & 9606.ENSP00000236850 & APOA1 & 25 \\
\hline 9606.ENSP00000360281 & $\mathrm{C} 8 \mathrm{~B}$ & 12 & 9606.ENSP00000241052 & CAT & 23 \\
\hline 9606.ENSP00000255040 & APCS & 10 & 9606.ENSP00000270142 & SOD1 & 23 \\
\hline 9606.ENSP00000415941 & $\mathrm{C} 4 \mathrm{~B}$ & 10 & 9606.ENSP00000378669 & ALDOA & 22 \\
\hline 9606.ENSP00000378130 & CFI & 10 & 9606.ENSP00000299767 & HSP90B1 & 22 \\
\hline
\end{tabular}

vesicle transport, and maintenance of cell activity. Interactions of aldolases $\mathrm{A}$ and $\mathrm{C}$ in light neurofilament (NF-L) expression may be linked to regulatory pathways that maintain the highly asymmetrical form and function of large neurons [29].

Some antioxidases such as catalase (CAT) and superoxide dismutase [Cu-Zn](SOD1) were in the downregulated group, which was consistent with the result found by Guler et al.'s research group [30]. Decreased activity of CAT and SOD1 in the plasma suggests a reduced antioxidant defense capacity in epilepsy patients. Protein disulfide-isomerase (P4HB), an endoplasmic reticulum chaperone, plays a crucial role in catalyzing disulfide bond formation, reduction, and isomerization. Kim et al. proved that protein disulfideisomerase is bound to NMDAR in the normal hippocampus and that this binding was increased in chronic epileptic rats. In addition, protein disulfide-isomerase knockdown effectively ameliorated spontaneous seizure activity in chronic epileptic rats. The finding suggested that protein disulfideisomerase might represent a target of potential therapeutics for epilepsy [31].

Heat shock protein $90 \mathrm{kDa}$ beta member 1(HSP90B1), a member of the Hsp90 family, showed decrease expression in the plasma of patients with epilepsy. Hong et al. discovered that HSP90B1 was a critical chaperone to integrate innate immunity, Wnt signaling, and organ development [32]. Di et al. reported that repressing the initial ER-associated degradation recognition step by inhibiting HSP90B1 enhanced the functional surface expression of misfolding-prone $\alpha 1(\mathrm{~A} 322 \mathrm{D})$ subunits of $\mathrm{GABA}_{\mathrm{A}} \mathrm{R}$, which caused autosomal dominant juvenile myoclonic epilepsy [33].

$\alpha$-Enolase (ENO1) was also in the downregulated group. In addition to glycolytic enzyme and plasminogen receptor functions, $\alpha$-enolase appears to have other cellular functions and subcellular localizations. As a plasminogen receptor, ENO1 binds to plasminogen and activates plasminogen conversion to plasmin. Ho-Tin-Noé et al. proved that limited plasminogen activation might contribute to nervous tissue organization during development, while uncontrolled plasmin formation might be neurotoxic [34]. Lahtinen et al. pointed out that the plasminogen system was involved in various events requiring extracellular proteolysis that were relevant to epileptogenesis, including cell migration and invasion, inflammation, and tissue repair and remodelling [35].

Apolipoprotein A1 (APOA1) was also found to be decreased in the plasma of patients with epilepsy. Apolipoprotein A1, a major component of high-density lipoproteins, has been shown to be involved in lipid metabolism, cholesterol homeostasis, and degeneration/regeneration of brain tissues and is proposed as a useful marker for the extent and severity of CNS injury. $\mathrm{Ni}$ et al. reported that in the model of neonatal SD rats induced by inhalation of flurothyl, the expression level of apolipoprotein A1 mRNA in the hippocampus of the recurrent epilepsy group was significantly decreased 30 days after the first seizure compared with the control group [36]. We speculate that the decreased level of apolipoprotein A1 in patients with epilepsy may indicate abnormal lipid metabolism and high risk of atherosclerosis.

However, there are some limitations of the study, such as the limited sample size. We have only included the minimum number of biological repeats required for proteomic analysis. But our study may provide clues for further study of large samples.

\section{Conclusions}

Our findings demonstrate that some differentially expressed proteins especially hub genes identified by TMT-based proteomics combined with bioinformatics analysis may be associated with epileptogenesis. Activation of acute-phase or innate immune response, complement and fibrinogen systems, and repression of glycolysis, lipoprotein metabolism, and antioxidant activity may play a role in the development of epilepsy. Some novel proteins are found out such as alpha-1-acid glycoprotein (ORM) and serum amyloid P-component (APCS). These new findings may provide novel insights into the pathogenic mechanisms of epilepsy and lead to a new therapeutic method in the future.

\section{Data Availability}

All relevant data are within the paper and its Supporting Information files. 


\section{Ethical Approval}

This study was carried under the approval of the Research Ethics Board of the First Hospital of Jilin University (Approval No. 2017-295).

\section{Consent}

Written informed consent was obtained from all the participants' parents or guardians prior to enrollment.

\section{Conflicts of Interest}

The authors declare that there is no conflict of interests regarding the publication of this paper.

\section{Acknowledgments}

We thank Ying Wang and Wenting Lu of the Institute of Pediatrics in the First Hospital of Jilin University for their input throughout the study. The present study was supported in part by grants from the National Nature Science Foundation of China (grant No. 81771396, No. 81171220, and No. 31371125 to JL); the Nature and Science Foundation from the Department of Science and Technology of Jilin Province (grant No. 20160101123JC to JL); the Fund of Pediatric Development from the First Hospital of Jilin University (JL); the Fund of Scientific Research Planning Project from the Department of Education of Jilin Province (grant No. JJKH20201048KJ to TJ and JS); and the Fund of Youth Science and Technology Training Project from Health Commission of Jilin Province (JS).

\section{Supplementary Materials}

Supplementary 1. Table S1: MS/MS spectrum database search analysis summary.

Supplementary 2. Figure S1: experimental strategy for quantitative proteome analysis and quality control validation of MS data. (a) Average peptide mass error. (b) Length distribution of all identified peptides.

Supplementary 3. Table S2: the list of identified protein between the epilepsy and control groups by TMT-based proteomics analysis.

Supplementary 4. Table S3: the list of differentially expressed protein between the epilepsy and control groups by TMTbased proteomics analysis.

\section{References}

[1] P. Iannetti, U. Raucci, L. A. Basile et al., "Benign epilepsy of childhood with centrotemporal spikes and unilateral developmental opercular dysplasia," Child's Nervous System, vol. 10, no. 4, pp. 264-269, 1994.

[2] N. L. Anderson and N. G. Anderson, "The human plasma proteome: history, character, and diagnostic prospects," Molecular \& cellular proteomics : MCP., vol. 1, no. 11, pp. 845-867, 2002.
[3] M. Seino, "Classification criteria of epileptic seizures and syndromes," Epilepsy research, vol. 70, Supplement 1, pp. 2733, 2006.

[4] M. Arnold, "Headache Classification Committee of the International Headache Society (IHS) The international classification of headache disorders, 3rd edition," Cephalalgia, vol. 38, no. 1, pp. 1-211, 2018.

[5] M. Kanehisa, S. Goto, M. Furumichi, M. Tanabe, and M. Hirakawa, "KEGG for representation and analysis of molecular networks involving diseases and drugs," Nucleic Acids Research, vol. 38, Supplement_1, pp. D355-D360, 2010.

[6] D. Szklarczyk, A. Franceschini, S. Wyder et al., "STRING v10: protein-protein interaction networks, integrated over the tree of life," Nucleic Acids Research, vol. 43, no. D1, pp. D447D452, 2015.

[7] M. S. Cline, M. Smoot, E. Cerami et al., "Integration of biological networks and gene expression data using Cytoscape," Nature protocols, vol. 2, no. 10, pp. 2366-2382, 2007.

[8] T. Ravizza, C. Kostoula, and A. Vezzani, "Immunity activation in brain cells in epilepsy: mechanistic insights and pathological consequences," Neuropediatrics, vol. 44, no. 6, pp. 330-335, 2013.

[9] N. Marchi, T. Granata, and D. Janigro, "Inflammatory pathways of seizure disorders," Trends in Neurosciences, vol. 37, no. 2, pp. 55-65, 2014.

[10] P. F. Fabene, G. N. Mora, M. Martinello et al., "A role for leukocyte-endothelial adhesion mechanisms in epilepsy," Nature Medicine, vol. 14, no. 12, pp. 1377-1383, 2008.

[11] W. Wang, L. Wang, J. Luo et al., "Role of a neural cell adhesion molecule found in cerebrospinal fluid as a potential biomarker for epilepsy," Neurochemical Research, vol. 37, no. 4, pp. 819$825,2012$.

[12] V. Iori, M. Maroso, M. Rizzi et al., "Receptor for advanced glycation endproducts is upregulated in temporal lobe epilepsy and contributes to experimental seizures," Neurobiology of Disease, vol. 58, pp. 102-114, 2013.

[13] A. M. Schmidt, S. Du Yan, S. F. Yan, and D. M. Stern, "The multiligand receptor RAGE as a progression factor amplifying immune and inflammatory responses," The Journal of Clinical Investigation, vol. 108, no. 7, pp. 949-955, 2001.

[14] P. Ambrogini, P. Torquato, D. Bartolini et al., "Excitotoxicity, neuroinflammation and oxidant stress as molecular bases of epileptogenesis and epilepsy-derived neurodegeneration: the role of vitamin E," Biochimica et Biophysica Acta Molecular Basis of Disease, vol. 1865, no. 6, pp. 1098-1112, 2019.

[15] E. Aronica, K. Boer, E. A. van Vliet et al., "Complement activation in experimental and human temporal lobe epilepsy," Neurobiology of Disease, vol. 26, no. 3, pp. 497-511, 2007.

[16] M. Kopczynska, W. M. Zelek, S. Vespa et al., "Complement system biomarkers in epilepsy," Seizure, vol. 60, pp. 1-7, 2018.

[17] C. Liguori, A. Romigi, F. Izzi et al., "Complement system dysregulation in patients affected by idiopathic generalized epilepsy and the effect of antiepileptic treatment," Epilepsy Research, vol. 137, pp. 107-111, 2017.

[18] N. D. Schartz, S. K. Wyatt-Johnson, L. R. Price, S. A. Colin, and A. L. Brewster, "Status epilepticus triggers long-lasting activation of complement $\mathrm{C} 1 \mathrm{q}-\mathrm{C} 3$ signaling in the hippocampus that correlates with seizure frequency in experimental epilepsy," Neurobiology of Disease, vol. 109, Part A, pp. 163173, 2018. 
[19] O. Unal, G. Deda, S. Teber, M. Ertem, and N. Akar, "Thrombophilic risk factors in epileptic children treated with valproic acid," Pediatric Neurology, vol. 40, no. 2, pp. 102-106, 2009.

[20] S. K. Wyatt, T. Witt, N. M. Barbaro, A. A. Cohen-Gadol, and A. L. Brewster, "Enhanced classical complement pathway activation and altered phagocytosis signaling molecules in human epilepsy," Experimental Neurology, vol. 295, pp. 184193, 2017.

[21] S. A. Hamed, "Atherosclerosis in epilepsy: its causes and implications,” Epilepsy \& behavior: E\& B, vol. 41, pp. 290-296, 2014.

[22] N. Cardenas-Rodriguez, B. Huerta-Gertrudis, L. Rivera-Espinosa et al., "Role of oxidative stress in refractory epilepsy: evidence in patients and experimental models," International Journal of Molecular Sciences, vol. 14, no. 1, pp. 1455-1476, 2013.

[23] A. B. Dixit, J. Banerjee, A. Srivastava et al., "RNA-seq analysis of hippocampal tissues reveals novel candidate genes for drug refractory epilepsy in patients with MTLE-HS," Genomics, vol. 107, no. 5, pp. 178-188, 2016.

[24] Z. Luo, H. Lei, Y. Sun, X. Liu, and D. F. Su, "Orosomucoid, an acute response protein with multiple modulating activities," Journal of Physiology and Biochemistry, vol. 71, no. 2, pp. 329-340, 2015.

[25] E. T. Poulsen, K. W. Pedersen, A. M. Marzeda, and J. J. Enghild, "Serum amyloid P component (SAP) interactome in human plasma containing physiological calcium levels," Biochemistry, vol. 56, no. 6, pp. 896-902, 2017.

[26] Z. Urbányi, L. László, T. B. Tomasi et al., "Serum amyloid P component induces neuronal apoptosis and beta-amyloid immunoreactivity," Brain Research, vol. 988, no. 1-2, pp. 6977, 2003.

[27] S. A. Hamed, E. A. Hamed, R. Hamdy, and T. Nabeshima, "Vascular risk factors and oxidative stress as independent predictors of asymptomatic atherosclerosis in adult patients with epilepsy," Epilepsy Research, vol. 74, no. 2-3, pp. 183192, 2007.

[28] M. SidAhmed-Mezi, R. Pumain, J. Louvel, P. Sokoloff, and J. Laschet, "New therapeutic targets to develop molecules active in drug-resistant epilepsies," Epilepsia, vol. 51, Supplement 3, pp. 43-47, 2010.

[29] R. Canete-Soler, K. S. Reddy, D. R. Tolan, and J. Zhai, “Aldolases a and $\mathrm{C}$ are ribonucleolytic components of a neuronal complex that regulates the stability of the light-neurofilament mRNA," The Journal of Neuroscience, vol. 25, no. 17, pp. 4353-4364, 2005.

[30] S. Keskin Guler, B. Aytac, Z. E. Durak et al., "Antioxidativeoxidative balance in epilepsy patients on antiepileptic therapy: a prospective case-control study," Neurological Sciences, vol. 37, no. 5, pp. 763-767, 2016.

[31] J. Y. Kim, A.-R. Ko, H.-W. Hyun, S.-J. Min, and J.-E. Kim, "PDI regulates seizure activity via NMDA receptor redox in rats," Scientific Reports, vol. 7, no. 1, p. 42491, 2017.

[32] F. Hong, S. Mohammad Rachidi, D. Lundgren et al., "Mapping the interactome of a major mammalian endoplasmic reticulum heat shock protein 90," PLoS One, vol. 12, no. 1, article e0169260, 2017.

[33] X.-J. Di, Y.-J. Wang, D.-Y. Han et al., “Grp94 protein delivers $\gamma$-aminobutyric acid type a (GABAA) receptors to $\mathrm{Hrd} 1$ protein-mediated endoplasmic reticulum-associated degradation," The Journal of Biological Chemistry, vol. 291, no. 18, pp. 9526-9539, 2016.
[34] B. Ho-Tin-Noe, H. Enslen, L. Doeuvre, J. M. Corsi, H. R. Lijnen, and E. Angles-Cano, "Role of plasminogen activation in neuronal organization and survival," Molecular and Cellular Neurosciences, vol. 42, no. 4, pp. 288-295, 2009.

[35] L. Lahtinen, K. Lukasiuk, and A. Pitkanen, "Increased expression and activity of urokinase-type plasminogen activator during epileptogenesis," The European Journal of Neuroscience, vol. 24, no. 7, pp. 1935-1945, 2006.

[36] H. Ni, Q. Sun, T. Tian, X. Feng, and B. L. Sun, "Long-term expression of metabolism-associated genes in the rat hippocampus following recurrent neonatal seizures and its regulation by melatonin," Molecular Medicine Reports, vol. 12, no. 2, pp. 2727-2734, 2015. 